\title{
Fortschritte in der Behandlung des
}

Diabetes mellitus.

Von Dr. J. Strauss in Frankfurt a. M.

(Fortsetzung aus No. 45.)

$$
\text { 2. Fette. }
$$

Nachdem festgestellt worden ist, daß die Acetonausscheidung eine Funktion gesteigerten Fettzerfalls ist, die bei dem Diabetiker auch 
bei Kohlehydratnahrung deshalb in Erscheinung treten kann, weil der Diabetiker die Kohlehydrate entweder garnicht oder. nur unvollkommen zu verbrennen vermag (Ge elmu y den (22), Wald vogel (107) Le o Schwarz (91), Hagenberg (108), H. Strauß und Philippsohn u. A.) bedurfte die Frage der klinischen Dignität der Fettzufuhr bei Diabetes einer Revision. Beobachtungen iiber den Einfluß der verschiedenen Fette auf die Acetonausscheidung bei Diabetes liegen vor von Leo S chwarz (93), Lépine (48), A. Löb (54), Grube (29) v. Noorden (76). Zusammenfassend wird das Thema behandelt durch v. Noorden und Schwarz (93). v. Noorden nimmt Stellung gegen die übertriebene Furcht ror einer durch Fett, insbesondere Butterfett drohenden Acidosis und macht auf folgende Punkte aufmerksam: Ein wesentlicher Einfluß der Fettzufuhr auf die Ausscheidung der Acetonkörper begiunt selbst in schweren Fällen erst, wenn die Fett-, beziehungsweise Butterzufuhr etwa $150 \mathrm{~g}$ pro die wesentlich übersteigt. Bei Verzicht auf diese Fettmenge würde zwar die Ausscheidung der Acetonkörper etwas vermindert werden, aber die Differenz ist nicht so groß, daß sie eine so tief in die Gesamternährung einschneidende Verordnung wie das Fettverbot rechtfertigen wïrde. Das Plus an Acetonkörpern wird bei fortgesetztem Alkaligebrauch leicht aus dem Körper entfernt, solange die Krankheit nicht in ein zügellos fortschreitendes Stadium getreten ist. Die Steigerung der Acetonkörper durch höhere Fettzufuhr markiert sich besonders in den ersten Zeiten hoher Fettzufuhr. Wenn der Fall aber in seiner Gesamtheit nur einigermaßen günstig liegt, sinkt nach 8-10 Tagen trotz hoher Fettzufuhr die Acetonausscheidung wieder auf die gleichen, oder niedrigere Werte als vor der Fettzufuhr. Hervorgehoben zu werden verdient auch die Tatsache, daß bei den Haferkuren v. Noordens trotz Zufuhr von $300 \mathrm{~g}$ Butter eine Abnahme der Acetonkörper zu verzeichnen war Lenné (46) spricht sich gleich v. Noorden gegen eine Beschränkung der Fettzufuhr in der Diabetikerdiät aus und empfiehlt, nach wie vor von der Darreichung der Fette den reichlichsten Gebrauch zu machen. Leo Schwarz, der die Mehrzahl der $\mathrm{Pa}$ tienten selbst große Fettmengen $(150-200 \mathrm{~g})$ trotz der Zunahme der Acetonurie ohne Störung des Allgemeinbefindens vertragen sah und der sich gleich v. Noorden davon ïberzeugte, daß. die anfängliche Zunahme der Acetonkörper bei fortgesetzter täglicher Aufnahme hoher Fettmengen im Laufe von ein bis zwei Wochen im allgemeinen abklingt, schließt sich dem Standpunkt v. Noordens insofern nicht vollkommen an, als er diejenigen Fälle, die übrigens nach seinem eigenen Urteil die Ausnahme von der Regel bilden, in denen es nach relativ geringer Fettzufuhr zll einer wesentlichen Vermehrung der Acetonurie kommt (zwei eigene Beobachtıngen von Schwarz, ein Fall von G. Kl emperer (38) gehören nach Schwarz gleichfalls hierher) generell durch genaue Kontrolle der Acetonurie während der Fettdarreichung berücksichtigt wissen möchte. Da die Kohlehydrate die Entstehung der Acetonkörper hemmen, empfiehlt Schwarz außerdem, gleichzeitig mit größeren Fettmengen nach Tunlichkeit Kohlehydrate zu verabreichen Was die Art der Diabetikern anzuratenden Fette anlangt, so plädieren v. Noorden und Schwarz dahin, der Butter, resp. dem Milchfett (dicker Rahm, fette Käsesorten) trotz des stärkeren Einflusses auf die Acetonausscheidung gegenïber dem Schweine- und Rinderfett und anderen Fettarten mit geringem Gehalt an flüchtigen Fettsäuren mit Rücksicht auf den Geschmack den Vorzug zu geben. v. Noorden(78) rät, die Butter gut auszuwaschen, wodurch ihr ein großer Teil der acetonvermehrenden Fettsäuren entzogen wird. v. Noorden (78) weist außerdem auf die Bedeutung des Eidotters hin. Löhning(55) hat sich inzwischen aus der Naunynschen Klinik bezüglich der nach reichlicher Fettsäurezufuhr auftretenden Acidose ähnlich dahin geäußert, daß wir noch garnicht wissen, ob diese ebenso ängstlich zu beurteilen ist wie die Acidose, die oftmals bei freier Diät zum Coma führt. L öhning führt zum Beweise dessen, daß man in der Ernährung des Diabetikers dieFette nicht unter das ïbliche Maß zu beschränken brauche, zwei Fälle von schwerem Diabetes an, in denen die Acidose bei gleichzeitiger Beschränkung der Eiweiß- nnd Fettzufuhr sehr viel stärker herunterging, als der Beschränkung der F'ettzufuhr allein entsprach. Das Sinken der Acidose ging in den beiden Fällen parallel mit der Abnahme der Zuckerbildung aus Eiweiß. Zu ähnlichen Schlüssen ist in der Klinik v. Merings Hübner (34) gelangt, insofern er es für zweckmäßig erachtet, „dem schweren Diabetiker den fïr den Organismus nötigen Kohlenstoff möglichst in Form von Fett zuzuführen, das wenn der Verdauungsapparat es zuläßt, in den größten Mengen genossen werden darf, während die Zufuhr von Eiweiß auf das geringste die Erhaltung des Eiweißbestandes im Körper noch ermöglichende $\mathrm{Ma}$ beschränkt werden muß“.

\section{Eiweißsubstanzen.}

Daß die diabetische Glycosurie durch Eiweißzufuhr gesteigert wird, ist von allen Autoren klar ansgesprochen worden (Bouchardat Cantani, Naunyn, v. Mering, v. Noorden). Dem entsptechend haben diese Autoren darauf hingewiesen, daß den Diabetikern nicht bloß die Kohlehydrate, sondern auch die Eiweißsubstanzen quantitativ zugemessen werden müssen. Cantani(12) erachtet $500 \mathrm{~g}$ gekochtes Fleisch als vollkommen ausreichend für alle Diabetiker. N a un yn, $(71,72)$ der die Beobachtung gemacht hat, daß einzelne Diabetiker nur dann Zucker ausscheiden, wenn sie sehr viel Fleisch genossen hatten, dagegen bei geringerem Fleischkonsum zuckerfrei werden, und der beim Diabetiker nichts dringender vermieden wissen will als eine Ueber lastung des Gesamtstoffwechsels, fixiert bei seiner „leichten gemischten Diät", bei der die fortgesetzte und genaue quantitative Regelung der Kost aufhört, gleichfalls die Eiweißmenge auf $500 \mathrm{~g}$ gekochtes Fleisch (alle Eiweißsubstanzen der Nahrung als Fleisch umgerechnet). Ungefähr dieselbe Menge ist im allgemeinen bei der "strengen" und "leichten" Fleischdiät Naunyns gestattet, bei welch letzterer das Nahrungsbedürfnis zum Teil durch Kohlehydrate gedeckt werden soll. In schweren Fällen von Diabetes verringert $\mathrm{N}$ aunyn die Eiweißnahrung allenfalls auf 200, resp. $150 \mathrm{~g}$ gekochtes Fleisch (entsprechend $40 \mathrm{bis} 50 \mathrm{~g}$ Eiweiß) und läßt da, wo die Zuckerausscheidung schon auf ein Minimum angelangt ist, zwecks Erzielnng der Aglycosurie - nach dem Vorschlag Cantanis - eventuell den Kranken 24 Stunden lang hungern. Aehnlich den Vorschriften Naunyns sind die Angaben v. Merings (61), der gleichfalls die Menge des bei Diabetikern zulässigen Eiweißes mit $500 \mathrm{~g}$ gekochtem Fleisch $(=700 \mathrm{~g}$ roh gewogen) normiert. Nach v. Noorden $(73,78)$ wird man bei leichteren Glycosurien bezüglich der Höhe der Eiweißzufuhr meistens über das für den Gesunden giiltige Durchschnittsma $\beta$ etwas hinauskommen und etwa 120 bis $140 \mathrm{~g}$ bei Erwachsenen, zirka 90 bis $100 \mathrm{~g}$ Eiweiß bei Kindern und Greisen geben. Für die Fälle von Diabetes, in denen nur mit großer Mühe oder überhaupt keine Zuckerfreiheit erzielt werden kann, empfiehlt v. Noorden Eiweißmengen von 70 bis $90 \mathrm{~g}$ pro die, deren durchschnittliche Zufuhr eventuell in der Weise durchzuführen ist, daß mehrwöchige Perioden, in denen $100 \mathrm{bis} 120 \mathrm{~g} \mathrm{Ei}$ weiß gestattet werden, mit solchen abwechseln, in denen die Eiweißzufuhr auf 50 bis $70 \mathrm{~g}$ eingeschränkt wird. Fïr die Unterbringung des letztgenannten Eiweißquantums eignen sich besonders die "Gemüsetage“ v. Noordens, an denen nichts anderes als grüne Gemüse mit seh viel Butter und Speck, Sesamöl oder Lebertran, Kaffee, Tee, Alcoholica verabreicht werden und bei denen der Eiweißumsatz auf 50 bis $70 \mathrm{~g}$ absinkt.

Eine gewisse gegensätzliche Stellung zu den genannten Autoren nimmt Talma (103) insofern ein, als el darauf aufmerksam macht, daß er die Arbeitsfähigkeit vieler Diabetiker zunehmen sah, als ganz enorme Eiweißmengen genommen wurden, und daß es ihm in zwe Fällen gelungen ist, die Acetonämie bei schwerem Diabetes durch Fütterung mit sehr viel Eiwei $\beta$ zum Verschwinden zu bringen. Letztere Beobachtungen gewinnen an Interesse dadurch, daß v. Noord en für die bei dem Coma diabeticum zu befolgende Diät den Rat erteilt, prinzipiell die Ernährungsform der vor dem Coma innegehaltenen entgegenzusetzen und, falls vorher die Kohlehydrate stark vertreten waren, allmählich zll einer kohlehydratarmen Eiweiß-Fettdiät überzugehen. v. Noorden hat unter Befolgung dieses Grundsatzes „in unmittelbarem Anschluß an ein Coma nachhaltige Erfolge erzielt" Immerhin bemißt Talma die den Diabetikern zuzuteilende Eiweißmenge gleich den anderen Autoren im allgemeinen gering, wenn er für die Eiweißzufuhr den N-Gehalt des Harns zur Grundlage nimmt und an erster Stelle dafür Sorge getragen haben will, daß die Stickstoffausscheidung die Einnahme nicht übertrifft, was man im allgemeinen erreiche, wenn das tägliche $\mathrm{N}$-Quantum \pm 16 betrage. Nur betrachtet Talma neinen großen N-Gehalt des Harns bei wenigen schweren Diabetikern bei geeigneter Behandlung als nachteilig, sei es auch, daf die Glycosurie dadurch zunehmen solle. Nachteilig ist es, wenn Albuminurie und Oxalurie dadurch hervorgerufen werden.

Man hat neuerdings der Höhe der Eiweißzufuhr bei leichteren Formen des Diabetes besondere Beachtung geschonkt.

Nach den Angaben von Kolisch (39), welcher die beim Diabetiker nach Eiweißzufuhr anftretende Glycosurie im Sinne einer unter der Reizwirkung der Eiweißnahrung auf die Gewebe zustande kommenden gesteigerten Znckerabspaltung auffaßt, „läßt sich in vielen Fällen zeigen, daß Diabetiker geringe Mengen von Kohlehydraten besser vertragen als die Zufuhr großer Mengen von Eiweiß“. K ol isch empfiehlt, um die Reizwirkung der Nahrung anf die Gewebe herabzusetzen unter Berufung auf die Untersuchungen von Weintraud (110), der bei einem Diabetiker mit 25 Kalorien pro Kilo Körpergewicht Stoffansatz erzielen konnte und auf ähnliche Befunde von Pautz -, eine Diät, die den Organismus mit einem Mindestmal3 von Kalorien im Gleichgewicht hält, und führt als'Vorteil des von ihm für die Behandlung des Diabetes vorgeschlagenen vegetarianischen Regimes an, daß dasselbe die Zufuhr großer Mengen von Nahrung gestatte, ohne den Organismus zu überernähren, ferner, daß man dem Organismus mehr Kohlehydrate zuführen könne, als dies neben der Fleischdiät möglich sei. Nach den Ausführungen Kolischs (40) hat der Diabetiker eher ein geringeres Ausmaß an Kalorien notwendig als der Gesunde, eine Erscheinung, die Kolisch als einen Selbstschutz des Organismus auffaßt, der sich bei den ungünstigen Ernährungsbedingungen, in welche der diabetische, zumal der schwer diabetische Organismus versetzt wird, auf ein geringeres Maß von Nahrung einstellt. Kolischs Empfehlung der Zufuhr einer kalorienarmen Nahrung steht in gewisser Hinsicht die Angabe Naunyns (71) zur Seite, der es "für ausgemacht 
hält, daß3 der Diabetiker mit einer Nahrung, welche einen viel geringeren Kalorienwert repräsentiert", als man nach dem für den erwachsenen normalen Menschen nach $R u b n e r$ berechneten Kalorienbedürfnis annehmen sollte, „durch Jahre auskommen kann, d. h. sich wohl befinden, geringe Arbeit leisten nnd dabei auf gleichem Körpergewicht verharren oder sogar an Gewicht zunehmen kann". Immerhin hat W. Schesinger (88) in seinen Untersuchungen bei Vergleich von Nahrungsaufnahme und Körpergewicht ein auffallend geringes Nahrungsbedürfnis des Diabetikers in Fällen von unkompliziertem Diabetes nur bei heruntergekommenen Kranken mit hoher Zuckerausscheidung feststellen können, also in Fällen, in denen man mit der Einschränkung der Eiweiß3nahrung auch bisher nicht gekargt hat. Der von Kolisch zitierte Fall Weintrauds betraf einen sehr schweren Diabetiker, und in den drei Fällen von $\mathrm{Pautz}$, in denen bei einer Kalorienzufuhr von 39,8-35,4 pro Kilo Körpergewicht Stickstoffansatz erfolgte, handelte es sich gleichfalls um schwere Diabetiker. Die Fälle, mit denen neuerdings Kolisch und Leclercq (41) die Annahme, daß die Einschränkung der Eiweißzufuhr den wesentlichen herabsetzenden Einfluß anf die Glycosurie übe, während die Mehr- oder Minderzufuhr von Kohlehydraten in ziemlich weiten Grenzen nur sekundär in Betracht komme, zu stützen suchen, betrafen ebenfalls schwere Diabetiker. Kolisch selbst geht übrigens einer schematischen Indikationsstellung für eine kalorienarme vegetarianische Kost aus dem Wege, indem er verlangt, daß in jedem einzelnen Fall das Nahrungsbedürfnis des Diabetikers, das v. Noorden im Gegensatz zu Kolisch nicht herabgesetzt fand, zu prüfen ist. $\mathrm{Ob}$ bei weiteren Untersuchungen leichtere Fälle von unkompliziertem Diabetes mit anffallend geringem Nahrungsbedürfnis sich herausstellen werden - und dieser Punkt erscheint mit Rücksicht auf die praktisch-therapeutische Seite des Gegenstandes nicht ohne Wichtigkeit - muß zunächst eine offene Frage bleiben.

Daß3 es leichtere Formen von Diabetes gibt, in denen es ohne Herabsetzung der Kohlehydratzufuhr lediglich durch Verminderung der Eiweißkost gelingt, den Harn zuckerfrei zu machen, ist gleich Kolisch auch Lenné (45) und v. Noorden (76) aufgefallen. v. Noorden präzisiert - unter Anführung eines besonders prägnanten Beispieles -

seine Erfahrungen dahin, dalß die Toleranz für Kohlehydrate bei hoher Eiweißzufuhr auch in leichten Fällen viel geringer ist als bei niedriger Eiweißzufuhr. "Es scheint, dab man durch fortgesetzt sehr hohe Eiweilgaben die Toleranz für Kohlehydrate dauernd schädigen kann." Auch aus der Naunynschen Klinik wird Aehnliches berichtet, indem Löhning (55) die Bemerkung macht: "Wir beobachteten häufiger, daß Diabetiker, die bei reichlicher Diät nicht zuckerfrei zu bekommen waren, bei sehr knapper Diät sogar geringe Mengen von Kohlehydraten vertrugen", und darauf hinweist, daf3 Naunyn diese Ansicht schon lange vertritt. Hierher kann man auch Beobachtungen von Talm a (103) rechnen, der bei Personen, die an Diabetes mellitus oder jedenfalls an Glycosurie gelitten hatten und durch die Fortdaner der Glycosurie veranlaß3t wurden, die Kohlehydrate zu verringern und die Eiweißmengen der Nahrung zu vergrölern, neben Albuminurie und Cylindrurie eine Glycosurie feststellte. Sobald nun wenig Eiweißß und viel Kohlehydrate genommen wurden, verschwand die Albuminurie und die Glycosurie, für welch letztere Talma die Möglichkeit eines renalen infolge der Sekretion von znviel $\mathrm{N}$-haltendem Material bedingten Ursprungs in Erwägung zieht. Was die therapeutische Beachtung dieser Tatsachen anlangt, so tritt v. Noorden, ohne der von Lenné postulierten zahlenmäßigen Normierung der Eiweißzufuhr zuzustimmen Lenné hat vorgeschlagen, die Eiweißzufuhr so lange zu verringern, bis die Harnstoffausscheidung den von ihm angenommenen Normalwert $0,37 \mathrm{~g}$ pro $\mathrm{kg}$ Körpergewicht und 24 Stunden erreicht - dafür ein, dal3 man auch in leichteren Fällen von Diabetes die Eiweilßzufuhr in vernünftigen Grenzen halten soll. Lenné hat übrigens seine Angaben über die Höhe der bei Diabetikern zweckmäßigen Eiweißßufuhr unlängst selbst dahin erweitert, daß er weniger Wert auf die zahlenmäßige Fixierung der Normalgrößje des Eiweißnumsatzes gelegt wissen will als darauf, daß3 die Diät des Diabetikers - unter Erhaltung des Stickstoffgleichgewichts - nicht bloß bei den mittelschweren und schweren Formen des Diabetes, sondern bei allen Diabetikern auf den Minimalverbrauch beschränkt werde.

Was die Art der Diabetikern anzuratenden Eiweißsubstanzen anlangt, so hat die Tatsache, daß in zahlreichen Eiweißkörpern der Nahrung Kohlehydratgruppen in verschiedenen Mengen gefunden wurden, Veranlassung zu der Frage gege ben, inwieweit die Art des mit der Nahrung eingeführten Eiweißes bei Diabetikern von Bedeutung für die Höhe der Glycosurie sein könne. Die Resultate der klinischen und experimentellen Prüfung decken sich jedoch nicht ohne weiteres mit dem, was man, von der Annahme ausgehend, daß die Höhe der nach Zufuhr verschiedener Albuminate auftretenden Glycosurie in erster Linie durch die in ihnen vorgebildeten Kohlehydratmoleküle bedingt werde, allenfalls erwarten konnte. L ï thj e $(57,58)$ sah bei einem Diabetiker nach Verabreichung des kohlehydratfreien Kaseïns eine stärkere Glycosurie als nach Rindfleisch-,Eiereiweiß-und Kalbsthymusnahrung. Bei Rindfleischnahrung war die Glycosurie höher als nach Eiereiweißnahrung. In Versuchen von Stradomsky (97) gab zwar Plasmon in einem schweren Falle von Diabetes die geringste Zuckerausscheidung, in dem mittelschweren Falle von Jiabetes war jedoch die Glycosurie nach Plasmon größer als nach Fleischnahrung. Bei Fleischnahrung wurde in dem schweren Falle von Diabetes mehr Zucker ausgeschieden als bei Zufuhr von Kalbsthymus, in dem mittelschweren Falle weniger. Beim Pancreasdiabetes des Hundes fand A. Berger (3) bei abwechselnder Fütterung mit Plasmon, Kalbsthymus und Fleisch das Verhältnis $\mathrm{N}$ : D ziemlich konstant. Falta (19), der auf die Beziehungen zwischen der Beeinflussung der Glycosurie durch die Albuminate und ihrer Zersetzlichkeit im Organismus aufmerksam macht, wie sie in der rascheren oder langsameren Elimination des aus ihnen stammenden Harnstickstoffs zum Ausdruck kommt, fand den Einfluß der von ihm unterSuchten Eiweißkörper dagegen sehr verschieden. Nach Kaseïn ging in seinem Falle die Zuckerausscheidung bedeutend in die Höhe, während eine Beeinflussung der Glycosurie durch Ovalbumin und Blutglobulin nicht zu erkennen war. Ferner gelang es, durch Ausschaltung des Eidotters ans der Kost, bei gleichzeitigem Ersatz durch eine entsprechende Menge von Muskelfleisch, den Patienten dauernd zuckerfrei zu machen. Neuerdings fand Falta (20), daf die Verabreichung von koaguliertem Eiereiwei $\beta$ im Gegensatz zum genuinen Eiereiweiß zu einem steilen Anstieg der N-Kurve und zu einer nicht unbeträchtlichen Vermehrung der Zuckerausscheidung führt. In Versuchen Lennés (46) mit Gelatine und Käse war trotz rigorosester Lebensweise des Kranken während vier, respektive fünf Tage - nicht der geringste Einfluß auf Verminderung der Zuckerausscheidung bemerkbar. SchumannLecle rcq (90) prüfte bei sieben Diabetikern den Einfluß von Roborat auf die Glycosurie im Vergleich zu Fleisch- und Käsenahrung. Von 36 Versuchsperioden sprachen 28 zugunsten des Pflanzeneiweißes. Immerhin waren die Differenzen oft recht gering, und nicht ganz selten gestaltete sich sowohl die absolute Zuckermenge wie auch der Quotient $\mathrm{N}: \mathrm{D}$ gerade in den Roboratperioden ungünstiger. v. Noorden (76) stellte als durchschnittliches Resultat seiner an mehr als $20 \mathrm{Dia}-$ betikern gemachten Beobachtungen folgende Skala für die Einwirkung der einzelnen Albuminate auf die Glycosurie auf: Am wenigsten führt Eierklar zur Glycosurie, dann folgen - ohne wesentliche Verschiedenheit voneinander - Pflanzeneiweiß, Kaseîn und die natürliche $\mathrm{Mi}$ schung von Eierklar mit Eigelb, wie sie in den Hühnereiern vorhanden ist, hierauf folgt in einigem Abstand das Muskeleiweiß, wobei ein Unterschied zwischen den verschiedenen Provenienzen nicht zu erkennen war. Für die Zwecke der praktischen D iätetik ist bemerkenswert, daß sich in den Versuchen v. Noordens die Individualität des Patienten als viel mal3gebender für die Bestimmung der Höhe der nach verschiedenen Albuminaten auftretenden Glycosurie herausstellte als die Eigenart der Eiweißkörper. So war mehrfach zu konstatieren, daß die Patienten auf den Austausch von Fleisch- und Eierkost oder von Fleisch und Roborat gar nicht reagierten. In vielen Versuchen stellte sich ferner das beachtenswerte Verhalten heraus, daß die Glycosurie sich verhältnismäßig günstig gestaltete, wenn nur ein è in zelner Eiweißkörper gegeben wurde, also z. B. nur Fleisch oder nur Eierklar etc. v. Noorden rät nicht dazu, aus den letztgenannten Untersuchungen für die Praxis größere Konsequenzen zu ziehen, einerseits, da - außer bei Gewährung der Fleischnahrung - jede andere einseitige Eiweißnahrung mit Rücksicht auf die Ansprtiche des Magens nur für kurze Zeit gereicht werden kann, andererseits, da der praktische Gewinn für die Verringerung der Glycosurie in keinem Verhältnis zu den Schwierigkeiten der Durchführung derartiger Diätbeschränkungen stehen würde. Dagegen will v. Noor de n auf die Tatsache, daß reines vegetabilisches Eiweil, z. B. Roborat, und Eier weniger ungünstig auf die 'T'oleranz einwirken als Fleisch, um so mehr Rücksicht genommen wissen, je schwerer die Glycosurie ist, aber immer unter besonderer Prüfung des im Einzelfall geltenden Verhaltens (78). - Für die Bewertung der einzelnen Diabetikerbrote dürfte neben den übrigen in Betracht kommenden Faktoren (Kohlehydratgehalt, Geschmack etc.) mit Rücksicht auf die günstigen durch v. Noorden und Schumann-Leclercq in übereinstimmender Weise mit Pflanzeneiweil3, respektive Roborat gemachten Erfahrungen auch die Frage, inwieweit ihnen ausschließlich Pflanzeneiwei $\beta$ und nicht Eiweiß anderer Provenienz beigemengt ist, von Belang sein. (Rademanns Diabetikerbrot [Pflanzeneiweiß $\beta]$, Gumperts Roboratgebäck, Aleuronatbrot.)

\section{Medikamentöse Therapie.}

Von der medikamentösen Therapie des Diabetes ist nach allem, was zurzeit darüber bekannt ist, in bezug auf eine Steigerung der Toleranz für Kohlehydrate nicht viel zu erwarten. Auf keinen Fall sind ihre Leistungen mit den Erfolgen der zielbewußten diätetischen Therapie in dieser Richtung auch nur annähernd zu vergleichen oder sind die Medikamente gar dazu berufen, an deren Stelle zu treten. Auch in anderer Hinsicht haben sich medikamentöse Verordnungen, denen gegen die diabetische Stoffwechselstörung als solche eine Wirkung zugeschrieben wurde, teils nicht bewährt, teils ist ihr Erfolg angezweifelt worden, teils kann das vorliegende Tatsachenmaterial nicht als genügend für die weitere Empfehlung angesehen werden. - Eine Ausnahme hiervon macht die Alkalibehandlung der diabetischen Acidosis, die für die Aufhaltung des Comas um so größere Chancen darbietet, 
je früher man mit ihr beginnt, je länger man sie fortsetzt (Stadel. mann, Naunyn, Magnus-Levy) (50) und je größere Dosen man anwendet (Naunyn, Magnus-Levy, v. Noorden).

$\mathrm{Naunyn}(71)$ äußert sich dahin, daß es „kein einziges Medikament außer dem Opium gibt, welches einigermaßen zuverlässig eine günstige Wirkung auf die Glycosurie oder die diabetische Stoffwechselstörung ausübt. Die angeblich durch die zahlveichen anderen Mittel erreichten Erfolge beruhen zum Teil daranf daß entweder unter dem Gebrauch der betreffenden Medikamente die Nahrungsaufnahme beschränkt oder die Resorption gestört wird. Sofern ein Mittel dies bewirkt oder dies gleichzeitig mit seiner Anwendung aus irgend einem Grunde geschieht, ist eine Herabsetzung der 'Zuckerausscheidung selbstverständlich und beweist nichts." Die Wirkung des Opiums, dessen Einfluß auf die Stoffwechselstörung $\mathrm{Naunyn}$ in Fällen von "reinem", d. h. nicht durch eine Erkrankung des Nervensystems kompliziertem Diabetes öfters nachweisen konnte, ist im übrigen, wie $\mathrm{Naun}$ yn und andere Autoren hervorheben, keine sichere und häufig keine dauernde, d. h. sie läßt nach, während das Mittel gebraucht wird. Einer breiteren Anwendung des Opiums steht schon die Tatsache entoegen, daß das Mittel sich zu dau erndem Gebrauch absolut nicht eignet. Dasselbe ist wohl auch, wenn auch nicht in demselben Maße, beziiglich der Salicylpräparate der Fall, für die neuerdings v. Noorden (73) (Aspirin), Williamson (115, 116) (Natr. salicyl. Aspirin), Zaudy (119) und Teschemacher (104) (Salol) in gewissem Sinne eintreten, im Gegensatz zu Eichhorst (17) und Litten (53), welch' letzterer das Natron salicylicum zwar für ein wirkungsloses Antidiabeticum, dagegen für ein Mittel von bedeutender symptomatischer Wirkung bei gewissen diabetischen Symptomen und Komplikationen erklärt.

Das von französischen Klinikern in die Therapie des Diabetes eingeführte Antipyrin bewährte sich F. Hirschfeld (31) - entsprechend seiner von Opitz (81) hervorgehobenen Wirksamkeit bei Diabetes insipidus - in den Fällen, in denen bei großer Zuckerausscheidung eine beträchtliche Polyurie lästig fällt. Die Zuckerausscheidung stieg in einem Falle Hirschfelds unter Antipyrin bei gleich bleibender Nahrung von $136 \mathrm{~g}$ auf $148 \mathrm{~g}$. v. Mering (61) fand das Antipyrin wirkungslos.

Von den Jambulpräparaten, auf deren Zweckmäßigkeit in der jüngsten Zeit Wilcox (114), Teschemacher, Smyth (96), Mackie (59) wieder hingewiesen haben, hat v. Noorden (73), der im allgemeinen verhältnismäßig Günstiges über sie berichtet, ,in einer Erfahrung, die sich auf mehr als 50 Fälle erstréckt, in keinem einzigen Falle von Diabetes eine von der Regelung der Diät unabhängige entscheidende Wirkung zum Guten gesehen". F. Hirschfeld (31) konstatierte gleichfalls nur eine sehr geringe Wirkung des Jambul. v. Mering (61) hat keine Erfolge mit Jambul erzielt.

Mit dem von A. Mayer (60) für die Behandlung des Diabetes vorgeschlagenen Sublimat hat v. Noorden (73) und ebenso in seinen noch weiter zu erwähnenden Untersuchungen Kaufmann (36) zwar in einigen Fällen einen deutlichen Einfluß auf die Zuckerausscheidung erzielt, doch überdauerte der günstige Einfluß des Medikaments auf die Zuckerausscheidung die Darreichung so kurze Zeit, daß v. Noorden es ablehnt, ein so gefährliches Medikament weiterhin in Anwendung zu bringen. Auch Hirschfeld (31) berichtet von schädlichen Nebenwirkungen des von ihm versuchsweise bei Diabetes angewandten Sublimats.

Die von englischer Seite empfohlenen Uransalze versagten v. Noorden (73) vollkommen, und Hirschfeld (31) sah in einem Falle schwere Verdauungsstörungen danach auftreten. Auch Monro (66) berichtet iiber einen Mißerfolg.

Die allenfallsige geringfügige Wirkung einiger in Laienkreisen beliebter Tees (Bohnenschalentee, Leinsamentee, Heidelbeerblättertee) auf die Zuckerausscheidung führt v. Noorden (73) - in Analogie mit dem Ausfall seiner noch zu erwähnenden Versuche mit heißem Karlsbader Wasser - auf den reichlichen Genuß sehr heißen Getränkes zurilck: In einem Versuche mit Leinsamentee fand v. Noorden unter dem Gebrauch des Tees eine geringe Vermehrung der Glycosurie. Umgekehrt ergab sich bei einem Versuche mit Bohnenschalentee eine ungefähr gleich große Verminderung der Zuckerausscheidung.

Literatilr:

34. K. Hübner, Zeitschrift für diätetische und physikalische Therapie, März 1904. 35. Jouls set, Semalne médicale 1896, S. 489. - 36. M. Ka u fm an n. Zeitschrift für klinische Medizin Bd. 48. - 37. K1rsteln, Theraple der Gegenwart 1899, No. 6. 38. G. Klemperer, Theraple der Gegenwart 1900. - 39. Kollsch, Wiener klinlsche Wochenschrift 1900, No. 52. - 40. Derseibe, Wiener medizinische Wochenschrift 1502, No. 20-22. - 41. Derselbe und Schumann-Leclercq, Wiener klinische Wochenschrift 1903 No 48. 42. Derselbe 1903, No. 48. - 42. Le Gendre, Gaz. des eaux 1898. Ref. in den Blättern für klinische Hydrotherapie 1898, No. 11.- 43. Le nn é, Therapeutische Monatshefte 1897, S. 244.44. Derselbe, Deutsche medizinische Wochenschrift 1898, Therapeutlsche Beilage, Oktober. - 45. Derselbe, Kongreß für Innere Medizin 1900 _- 46. Derselbe, Balneologenkongreß 1903 Berliner klinische Wochenschrift 1903 und Deutsche MedizInalzeltung 1903, No. 64. 47. L e o, Kongreß für innere Medizin 1898. -- 48. Lép i n e, Semaine médicale 1901, S. 361. - 49. Derselbe, Mitteilung über Hefetherapie. Zitiert nach M. Ka u fmann. - 50. A. Mag. n us - Lev y, Archiv für experinlentelle Pathologie und Pharmakologie 1899. - 51. Derselbe, Archiv für experimentelle Pathologie und Pharmakologie 1901. - 52. Li nossier, Lyon néd. 1900, No. 11. Ref. in Virchow-Hirschs Jahresberichten 1900, Bd. 2, S. 46. - 53. Litten, Therapie der Gegenwart 1899, No. 3. - 54. A. L $\measuredangle$ b, Zentralblatt für Stoffwechsel- und Verdauungskrankheiten 1902, No. 8. - 55. K. Lô hnlng, Theraple der Gegenwart, März 1904.
56. A. Lor and, Policlinique 1903, No. 20. Ref. im Zentralbtatt für Stoffwechsel- und Verdautungskrankheiten 1904, No. 5. -- 57. H. Lü th j e, Zeitschrift für klinische Medizin Bd. 39. - 58. Derselbe, Zeitschrift für klinische Medizln Bd. 43. - 59. W. Ma ck kie, British nledical journal 1901, Bd. 2, S. 618. - 60. A. Ma y e r, New York med. Records 1898. Zitier Ilach v, Noorden, Die Zuckerkrankhelt und thre Behandlung, Berlin 1901. - 61. v. Me r Ing, Pentzoldt-Stintzings Handbuch Bd. 2. -62 . Min kowski, Archlv für experimentelle Pathologie und Pharmakologie 1893, Bd. 31. - 63. Derselbe, Kongreß für innere Medizin 1898. - 64. Moh r, v. Noordens Sammlung klinischer Abhandungen uiber Patho Medizln Theraple der Stoffwechsel- und Ernährungsstörungen 1904 - 65. Mo h $\mathrm{r}$ und L $0 \mathrm{~b}$, Zentral-

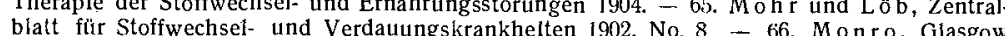

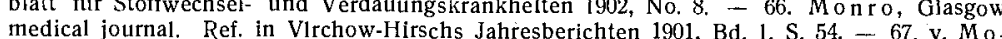
medical journal. Ref. in Vlrchow-Hirschs Jahresberichten 1901, Bd. 1, S. 54. - 67. v. Mo raczew s k1, Zeitschrift für klinische Medizin Bd. 34. Derselbe, Zentralblatt für innere Medizin 1897, No. 30. - 68. A. Mos s é, Sernaine médlcale 1896, S. 338. - 69. Derselbe, Comptes rend Bd. 133 , No. 24. -70 . Derselbe, Revue de médeclne, 22. Jahrgang 1902, No. 2 u. a. a. O. 71. N a uny n, Der Diabetes mellitus. Nothnagels spezielle Pathologie und Therapie Bd. 7 . Wien 1898. - 72. Der Diabetes mellitus. Deutsche Klinik 1901. - 73. C. v. Noorden, Die Zuckerkrankileit uud ihre Behandlung. Berlin 1901. - 74. Derselbe, Irrwege in der Behandlung der Zuckerkrankheit. Aerztlicher Ratgeber, 3. Jahrgang 1901. - 75. Derselbe, Deutsche Praxis 1901. -- 76. Derselbe, Wiener medizinische Presse 1902, No. 40. - 77. Derselbe, Berliner klinische Wochenschrift 1903, No. 38. - 78. Derselbe, Leydens Handbuch der Ernährungstherapie 1904. - 79. O ffer, Diskussion zul dem Vortrage von Kolisch: Ueber Dlabetesdiăt. Gesellschaft für innere Medizln in Wien, 3. Dezernber 1903. - 80. Oliver Lancet 1897 und 1898. Ref. in Virchow-Hirschs Jahresberichten. - 81. M. O pit $z$, Deutsche medizinische Wochenschrift 1889, No. 32. - 82. O ser, Die Erkrankungen des Pancreas. Nothnagels spezielle Pathologle und Therapie Bd. 18. - 83. P a u tz, Zeitschrift für Biologie 1895. - 84. Roget et Balvay, Lyon méd. 1899, No. 2 und 3. Ref. in Virchow-Hirschs Jahresberichten Bd. 2, S. 42, - 85. S. Ro se 11 berg, Deutsche Aerztezeitung 1902 N0.17 (Schluß folgt.) 